\begin{tabular}{cc}
\hline & International Journal of Engineering \& Technology, $7(4.30)(2018) 443-447$ \\
SPC & International Journal of Engineering \& Technology \\
& Website: www.sciencepubco.com/index.php/IJET \\
Research paper & \\
\hline
\end{tabular}

\title{
Analysis of Toothbrush Rig Parameter Estimation Using Different Model Orders in Real-Coded Genetic Algorithm (RCGA)
}

\author{
Ainul, H.M.Y. ${ }^{1}$, Salleh, S.M. ${ }^{*}$, Halib, N. ${ }^{2}$, Taib, H. ${ }^{1}$, Fathi, M.S. ${ }^{1}$ \\ ${ }^{I}$ Mechanical Failure Prevention and Reliability (MPROVE), Department of Engineering Mechanics, \\ ${ }^{I}$ Faculty of Mechanical and Manufacturing Engineering, Universiti Tun Hussein Onn Malaysia, \\ ${ }^{2}$ Faculty of Dentistry, Universiti Sains Islam Malaysia. \\ *Corresponding author E-mail:saliha@uthm.edu.my
}

\begin{abstract}
System identification is a method to build a model for a dynamic system from the experimental data. In this paper, optimization technique was applied to optimize the objective function that lead to satisfying solution which obtain the dynamic model of the system. Realcoded genetic algorithm (RCGA) as a stochastic global search method was applied for optimization. Hence, the model of the plant was represented by the transfer function from the identified parameters obtained from the optimization process. For performance analysis of toothbrush rig parameter estimation, there were six different model orders have been considered where each of model order has been analyzed for 10 times. The influence of conventional genetic algorithm parameter - generation gap has been investigated too. The statistical analysis was used to evaluate the performance of the model based on the objective function which is the Mean Square Error (MSE). The validation test-through correlation analysis was used to validate the model. The model of model order 2 is chosen as the best model as it has fulfilled the criteria involved in selecting the accurate model. Generation gap used was 0.5 has shorten the algorithm convergence time without affecting the model accuracy.
\end{abstract}

Keywords: Modeling; objective function; system identification; validation.

\section{Introduction}

Modeling dynamic system to simulate the actual behavior of the system based on the mathematical relationship of the input and the output is called as system identification. It involved a process of acquiring, processing and constructing mathematical models based on raw data obtained from the real system [4]. In this study, toothbrush rig which provide circular and forward backward motion is used as a real plant. The best model should simulate the dynamic behavior of the real plant in accurate condition.

The modeling process can be achieved through two conditions: the first condition is where the order and the parameters of the model might be fully known, and it can be all or partially of the parameters are unknown [1]. While, the second condition is where the orders and parameters of the process might be fully unknown which is called as black box model.

Important step for adequate modeling of dynamic models is the choice of optimization process for model parameter identification. The real-coded genetic algorithm (RCGA) will be used as optimization technique for parameter estimation process. Genetic algorithm as a stochastic global search method find the satisfying solution in complex multidimensional search spaces and evaluate many points simultaneously [2-3]. It has been reported, by using genetic algorithm as optimization method, the optimal solution can be achieved as it converges to optimal solutions by avoiding the local minima by several researches [5-8].

Parameter estimation process use genetic algorithm as the optimization technique with its objective is to minimize the error be- tween actual and predicted output. Validation test for the model is vital to validate either it is capable to produce the measured data or not. The accepted model can be selected by correlation analysis and percentage of model fitting [9].

In this paper, the objective that need to be achieved is estimate the parameter to have the best model which close to real plant. The data was acquired from the experiment by considering single input and single output. Real-coded genetic algorithm (RCGA) was applied as optimization method. The analysis of each six-different model order has been analyzed for ten (10) times. Also, the influence of conventional genetic algorithm parameter - generation gap has been considered. The performance of the model was evaluated based on the objective function optimization. The model is validated by auto-correlation and cross-correlation analysis.

\section{System Identification}

The modelling of dynamic system in this paper is constructed by using system identification. The mathematical relationship of input and output is based on the experimental data from the toothbrush rig experiment. There are few steps involved in obtaining the accurate model from the system identification. Steps in system identification are data acquisition, selecting model structure, parameter estimation and model validation. Real-coded genetic algorithm (RCGA) is chosen to be used as optimization method in the system identification process. It has an advantage in searching the best model as it can escape the local minima in converging to achieve the optimal solution. 


\subsection{Experimental design}

The experimental data to be used for system identification is acquired from the toothbrush experiment rig. The experiment is conducted from the toothbrush rig with complete component which provide circular and forward backward motion to simulate the actual behavior of brushing teeth. The schematic design of toothbrush experimental rig is shown in Fig. 1.

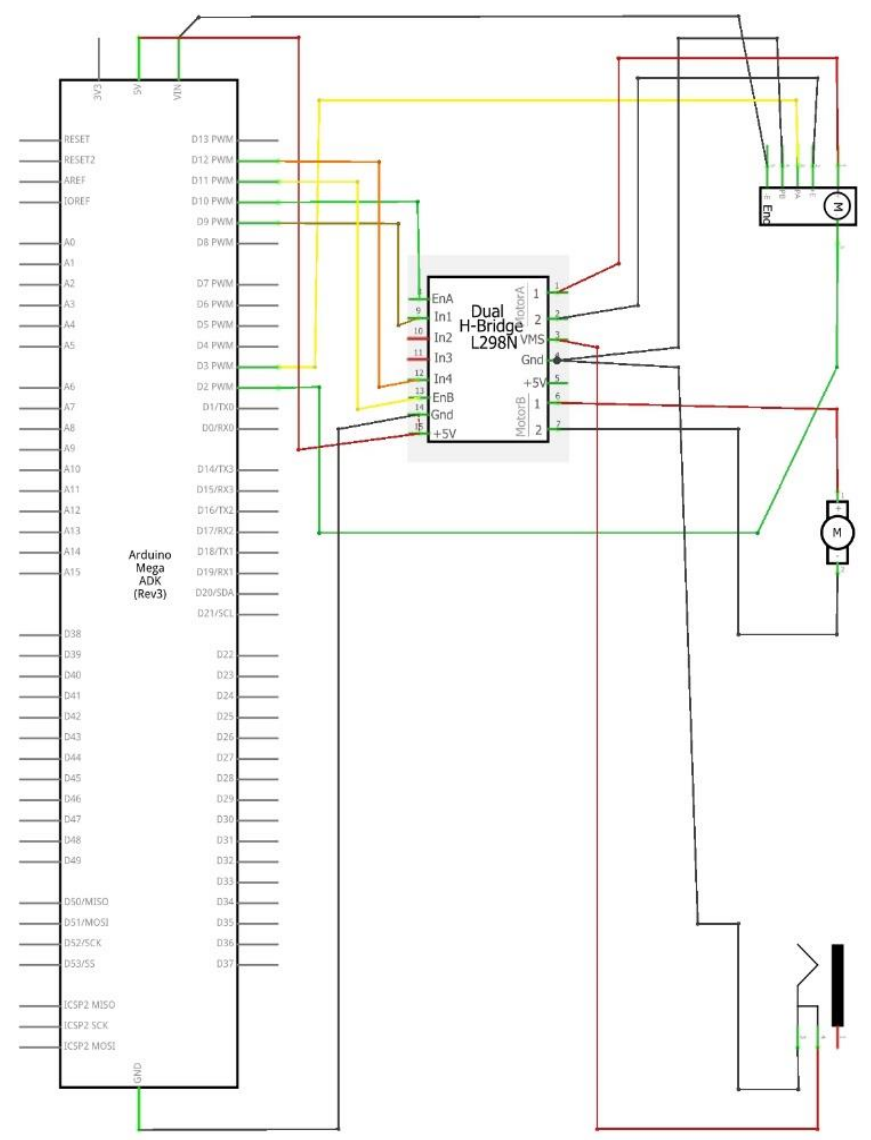

Fig. 1: Experimental design of toothbrush experimental rig.

The input was voltage supplied to the actuator and the output was the speed of the actuator which detected by the encoder. The experiment starts by setting the PWM value in the coding for the toothbrush experimental rig. The PWM value which indicate the voltage is supplied to the actuator from the motor driver. Speed of the actuator is captured by the encoder. The voltage and the speed of the actuator was measured as the experimental data. This data is the vital element in estimating the model parameter to construct the model that close to the real plant.

\subsection{Model structure}

Choosing model structure is required before parameter estimation. The model structure is chosen by understanding the characteristic or behavior of the variable voltage for the system. From the analysis of the experiment conducted on the system, the system is a nonlinear because of many disturbances acting on it. The single input single output (SISO) of dynamic model used for this analysis. In this study, autoregressive with exogenous input (ARX) model structure was chosen. The equation used to describe the system which is known as polynomial model is given by equation (1)

$$
A\left(z^{-1}\right) y(k)=B\left(z^{-1}\right) u(k)+\varepsilon(t)
$$

Where consist of equation (2) and (3)

$$
\begin{aligned}
& A\left(z^{-1}\right)=1+a_{1} z^{-1}+\ldots a_{n} z^{-n} \\
& B\left(z^{-1}\right)=1+b_{1} z^{-1}+\ldots b_{n} z^{-n} \\
& \varepsilon(t)=0 \text { (white noise) }
\end{aligned}
$$

Thus, ARX model also can be written as in (4)

$$
\begin{aligned}
y(k)= & -a_{1} y(k-1) \ldots-a_{n} y(k-n)+b_{1} u(k-1) \\
& +b_{n} u(k-n)+\varepsilon(t)
\end{aligned}
$$

The transfer function is obtained to represent the real system behavior. The best model is obtained after the analysis has been done for ten (10) times for each six-different model and optimized by using real-coded genetic algorithm (RCGA).

\subsection{Parameter estimation}

There are some parameters need to be estimated in obtaining the best model of a system. The accuracy parameter involved is important in prediction of the system performance. After constructing a model of the toothbrush rig by using ARX equation, the parameters will be identified by optimizing the objective function. Genetic Algorithm as the global search method is used to estimate the parameters required to form the best model.

\subsection{Objective function}

The performance of the model is validated by using statistical analysis. Genetic Algorithm is applied to optimize the model parameters by minimizing the objective function. The objective function taken in this study was the Mean Square Error (MSE) which defined by equation (5)

$M S E=\frac{1}{N} \sum_{i=1}^{N}\left(y_{i}(t)-\hat{y}_{i}(t)\right)^{2}$

where

$y_{i}=$ Actual output data

$\hat{y}_{i}=$ Predicted output data

The Mean Square Error (MSE) between actual and predicted output is measured to identify the parameters of the model. By minimizing the MSE value using the real-coded genetic algorithm (RCGA), the suitable parameters for the model can be identified. Hence, model parameters with the least value of MSE is considered as the best model parameters.

The pole-zero map of identified system is referred for validation process. Pole-zero plot is representing the transfer function in a graphical form that give an information of the stability and other characteristic of the model. Besides, correlation test also used to validate the dynamic model. Error signal is defined as the difference between normalized predicted output data and real output data. Two error signals are used to produce correlation signal for auto-correlation test. While, the error data is used to be correlated with the normalized input data to produce correlation signal for cross-correlation test.

\subsection{Real-Coded Genetic Algorithm (RCGA) procedure}

The next step after selecting the model structure was estimating the behavior of the system. There are many methods can be used in this step. In this study, Genetic Algorithm is used to estimate the parameter of the dynamic system in constructing the best model for the system. The purpose of implementing Genetic Algorithm toward a population of chromosome was to optimize the candidate chromosome toward better solution.

Hence, the first step of Genetic Algorithm is created the population consist of varies number which is called individual, represents 
the parameters of the dynamic model. The population making is based on the number of model order used. The performance analysis of the model in this study is based on the six-different model order with different generation gap value used to search the best model as stated in Table 1.

Table 1: Six-different model order with different generation gap value that used to analyze the performance of parameter estimation of ARX model.

\begin{tabular}{|c|c|}
\hline Generation Gap & \multicolumn{2}{|c|}{ Trial model order } \\
\hline 0.5 & \multirow{2}{*}{$2,3,4,5,6,7$} \\
\hline 0.7 & \\
\hline 0.9 & \\
\hline
\end{tabular}

Each model order has been analyzed for ten (10) times iteration with three different values of generation gap. Then, the fitness value of each chromosome is evaluated. The fittest individual will have a chance to be selected while the less fit individual is forced out from the population. Generation gap values will make the selection of the individual from the current population. The selected individuals are modified to create new generation by the crossover and being mutated. This step will be repeated and continue produced new generation to be used in the next iteration till the best parameter identified.

Compared to crossover and mutation, generation gap has been varied and the result in estimate the model parameter has been investigated in this study. The generation gap (GGAP) parameter in excluded from the others parameter because it can affect the performance of the optimization process. The chosen of GGAP parameter has the tendency of influence can be drawn. Compared with the parameters crossover (XOVR) and mutation (MUTR), has no tendency of influence on the optimization process.

All the conventional parameter of genetic algorithm involved in searching process; NIND-number of individuals, MAXGENmaximum generations number, NVAR-number of variation, number of trial for each model order with three different values of generation gap are set up before the starting the searching process. The value of each parameter used in this study is presented in Table 2 .

Table 2: Selected value for parameters involved in genetic algorithm.

\begin{tabular}{|c|c|}
\hline Parameter & Value \\
\hline NIND & 100 \\
\hline MAXGEN & 300 \\
\hline NVAR & equal to model order \\
\hline Number of Trial & 10 times \\
\hline
\end{tabular}

All the parameters in Table 2, will decided probability in searching process. The NIND value created the probability of selected individual to be selected in a population. The objective function of the genetic algorithm will be optimized to obtain the parameters of ARX model. The algorithm will terminate when the maximum generation is achieved.

\section{Results and Discussion}

The identification process of the system has been optimized by using Genetic Algorithm method. The performance analysis of toothbrush rig parameter identification is analyzed using different model orders in real-coded genetic algorithm (RCGA). This is because the model order effect the optimization of objective function. Model order value initiate the population for the searching process. It was related with the convergence graph of real-coded genetic algorithm (RCGA) in converging to the minimum objective function and terminate when reach maximum generation.

Model order of 4 and 6 for each generation gap, converge and be at the steady state around 80 generation from the 300 generation done. While, another model order only reaches steady state after 100 generation. Hence, the model order of 4 and 6 converge faster because of the order is getting close to the suitable parameter of the model. Also, the converging to minimize the objective func- tion with model order of 4 and 6 shows the constant objective function value for each generation gap. The objective function value of model order 4 and 6 are 0.020132 and 0.025115 which considered as the smallest value compared to another model order. Hence, both model orders are taken for further analysis in estimating the parameter of toothbrush rig.

The influence of Genetic Algorithm parameter-generation gap has been analyzed too as it gives an effect to the model estimation process. The analysis has been done for six-different model orders with different generation gap value. Each model order has been analyzed about 10 times by Genetic Algorithm to find the best parameter. The Mean Square Error (MSE) value of each model with different generation gap is shown in Fig. 2.

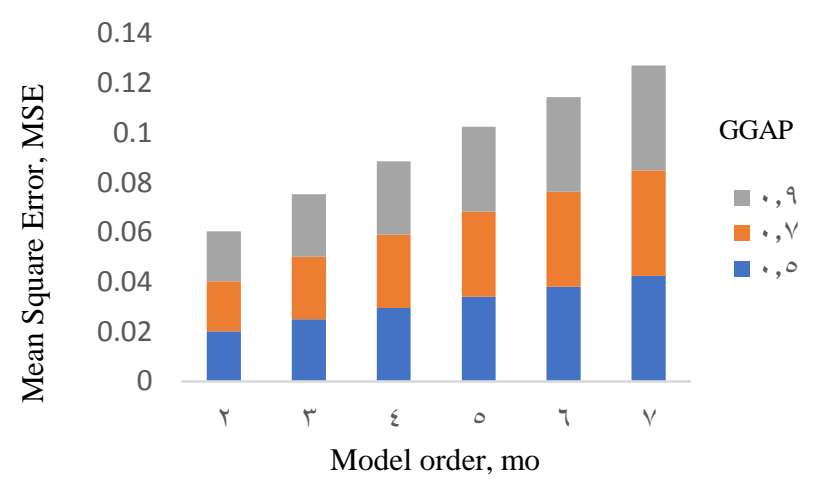

Fig. 2: Numerical data showing the Mean Square Error (MSE) obtained with six-different model orders and three values of generation gap.

Fig. 2 shows the optimization criterion values obtained for three different value of Genetic Algorithm parameter-generation gap obtained with six-different model orders. Model order 4 and 6 indicates the best model as both represent the least and accurate Mean Square Error value for each generation gap. The Mean Square Error (MSE) values obtained for each model order, increases with increasing model orders. The lowest model order gives simple equation, but it may not reliable to represent the real system, while the higher model order with complex equation may over fitting the real system.

Both model order was evaluated in the next step which is time execution. In this paper, the effect of the Genetic Algorithm parameter - generation gap was studied based on the performance of optimizing the objective function to achieve the optimal solution. The influence of generation gap value is observed in terms of the convergence time for ten (10) times analysis. The results of model parameter identification using different generation gap is stated in Table 3.

Table 3: Results of model parameter identification using different value of generation gap.

\begin{tabular}{|c|c|c|c|c|c|c|}
\hline & \multicolumn{6}{|c|}{ Generation Gap } \\
\hline & \multicolumn{2}{|c|}{0.5} & \multicolumn{2}{|c|}{0.7} & \multicolumn{2}{|c|}{0.9} \\
\hline 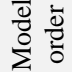 & 4 & 6 & 4 & 6 & 4 & 6 \\
\hline$\sum^{\sqrt[1]{n}}$ & $\begin{array}{c}0.0201 \\
3\end{array}$ & $\begin{array}{c}0.0251 \\
2\end{array}$ & 0.02013 & 0.02512 & 0.02013 & 0.02512 \\
\hline 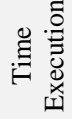 & $\begin{array}{c}51.074 \\
8\end{array}$ & $\begin{array}{c}51.204 \\
7\end{array}$ & $\begin{array}{c}119.453 \\
2\end{array}$ & $\begin{array}{c}125.183 \\
9\end{array}$ & $\begin{array}{c}159.127 \\
3\end{array}$ & $\begin{array}{c}150.904 \\
4\end{array}$ \\
\hline
\end{tabular}

Table 3 shows the optimization criterion values obtained with three kinds of generation gap values is constant for the same model order. The MSE values obtained with model order 4 and 6 are the same for three different generation gap values, but the conver- 
gence time of smallest generation gap is less than the highest generation gap. The constant MSE value obtained prove that the generation gap reduces the convergence time without loss of model accuracy.

The results of optimizing objective function with generation gap of 0.5 is distinguished as the fastest algorithm instead of 0.9. It shows that the generation gap is the most sensitive parameter concerning the convergence time. The last step to select the best model has been done by using decision matrix method. The criteria that has been discussed previously has been considered in this method. The decision matrix in choosing the best parameter is presented in Table 4.

Table 5: Decision Matrix of toothbrush rig parameter

\begin{tabular}{|c|c|c|c|c|c|c|c|c|c|c|c|c|c|}
\hline & & \multicolumn{12}{|c|}{ Generation Gap } \\
\hline & & \multicolumn{4}{|c|}{0.5} & \multicolumn{4}{|c|}{0.7} & \multicolumn{4}{|c|}{0.9} \\
\hline \multicolumn{2}{|c|}{ Model order } & \multicolumn{2}{|c|}{4} & \multicolumn{2}{|c|}{6} & \multicolumn{2}{|c|}{4} & \multicolumn{2}{|c|}{6} & \multicolumn{2}{|c|}{4} & \multicolumn{2}{|c|}{6} \\
\hline Criteria & $\begin{array}{c}\text { Weight score } \\
(\%)\end{array}$ & $\mathrm{a}$ & $\mathrm{b}$ & a & $\mathrm{b}$ & a & $\mathrm{b}$ & a & $\mathrm{b}$ & a & $\mathrm{b}$ & a & $\mathrm{b}$ \\
\hline MSE & 60 & 3.0 & 5 & 0.6 & 1 & 3.0 & 5 & 0.6 & 1 & 3.0 & 5 & 0.6 & 1 \\
\hline $\begin{array}{c}\text { Time } \\
\text { Execution }\end{array}$ & 40 & 2.0 & 5 & 1.6 & 4 & 1.2 & 3 & 0.8 & 2 & 0.4 & 1 & 0.4 & 1 \\
\hline Total score & 100 & \multicolumn{2}{|c|}{5.0} & \multicolumn{2}{|c|}{2.2} & \multicolumn{2}{|c|}{4.2} & \multicolumn{2}{|c|}{1.4} & \multicolumn{2}{|c|}{3.4} & \multicolumn{2}{|c|}{1.0} \\
\hline \multicolumn{2}{|c|}{ Rank } & \multicolumn{2}{|c|}{1} & \multicolumn{2}{|c|}{4} & \multicolumn{2}{|c|}{2} & \multicolumn{2}{|c|}{5} & \multicolumn{2}{|c|}{3} & \multicolumn{2}{|c|}{6} \\
\hline
\end{tabular}

*a represents the weight score of each criterion

*b represents the rating of each criterion

After considered all criteria as stated in Table 4, the best parameter has been selected. All the value of each criteria that obtained by Real-Coded Genetic Algorithm (RCGA) optimization method has been compared. The weight score for each criterion is based on the priority of the criteria for the selection process. The rating for each model based on criterion has been compared from the value stated in Table 4. Then the total score is calculated from the total of multiplication of weight score and the rating. The model with the highest score has been ranked at the first position and being elected as the best parameter for the model.

The small value of MSE means that the model has converged to the best objective function. This is because from the theory of genetic algorithm, the optimization process is to converge to the optimal solution. Hence, the succeed optimization process is based on the minimizing of the objective function which is referred to the MSE value. Standard deviation is obtained from the ten (10) times analysis. The smallest value of standard deviation makes the model order as the best model order. This is because it shows that the analysis for that model order from the first until ten analyses are constant and stable.

Generation gap is defined as the portion from the population that is replaced by the new offspring at each generation. The larger the value of generation gap used, the larger the portion of the population being replaced make the optimization process of the objective function became more difficult. Longer time is needed to optimize the objective function because the old chromosome in that population need more time to adapt with the large portion of the new chromosome that is replaced for each generation. Hence, the analysis with small generation gap value with short convergence time was the effective and efficient value for the optimization process.

Hence, all the criteria have been compared and the total score for each model order has been calculated. As a result, from the RealCoded Genetic Algorithm (RCGA) optimization method, model order of 2 produced the lowest prediction MSE value of 0.020132 with 51.0748 s convergence time. Generation gap used was 0.5 has shorten the algorithm convergence time without affecting the model accuracy. The selected parameter is shown in Table 5.

Table 5: Parameter identified from Real-Coded Genetic Algorithm (RCGA) optimization method.

\begin{tabular}{|c|c|c|}
\hline \multirow[b]{2}{*}{$\begin{array}{l}\text { Model } \\
\text { order }\end{array}$} & \multicolumn{2}{|c|}{$\mathrm{a}$} \\
\hline & $\mathrm{a} 0$ & $\begin{array}{l}\text { a1 } \\
\text { a2 }\end{array}$ \\
\hline \multirow{4}{*}{6} & -0.4548 & -0.1984 \\
\hline & \multicolumn{2}{|c|}{$\mathrm{b}$} \\
\hline & b0 & b1 \\
\hline & 0.4427 & -0.0553 \\
\hline MSE & \multicolumn{2}{|c|}{0.020132} \\
\hline $\begin{array}{c}\text { Time } \\
\text { Execution }\end{array}$ & \multicolumn{2}{|c|}{51.0748} \\
\hline
\end{tabular}

Therefore, the best model representing the real system is in transfer function written in the form of ARX equation, represented by equation (6).

$t f=\frac{0.4427-0.0553 z^{-2}}{1-0.4548 z^{-1}-0.1984 z^{-2}}$

The selected parameter of ARX model, has been validate by residual test. The auto-correlation should produce sharp spike at the center. All cross-correlation should be within the confident line. Auto-correlation is shown in Fig. 3 and Fig. 4, 5, 6 represents the cross-correlation.

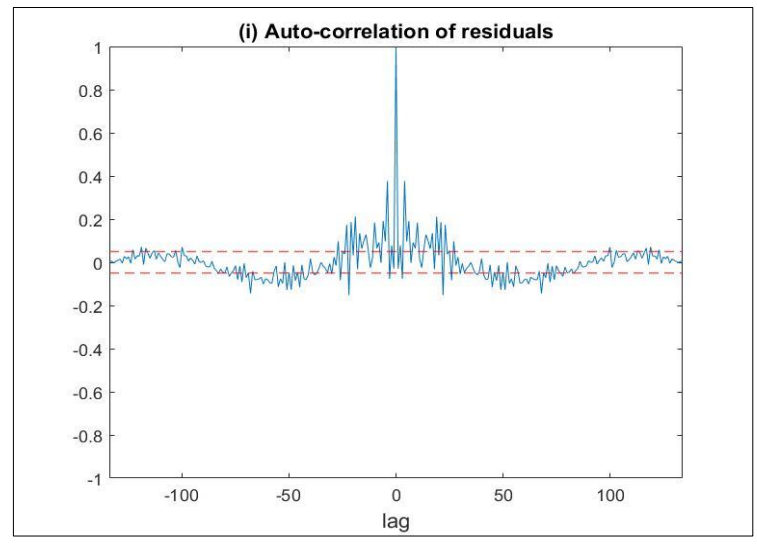

Fig. 3: Validation test with auto-correlation.

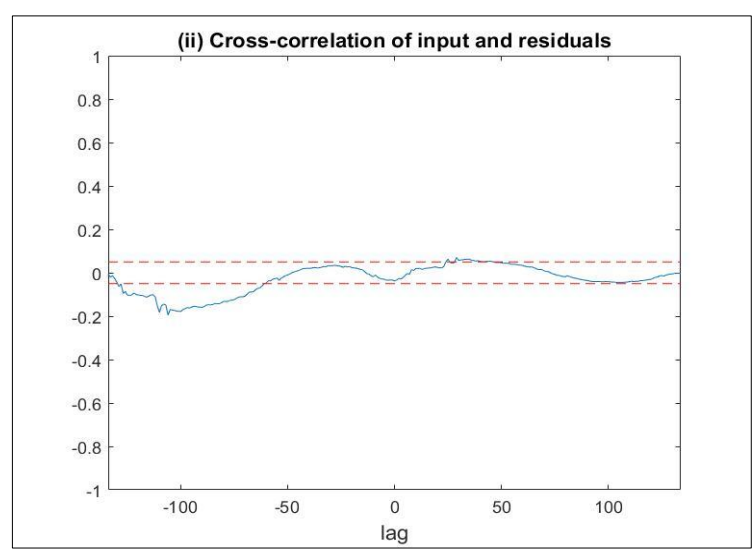

Fig. 4: Validation test with cross-correlation of input and residuals. 


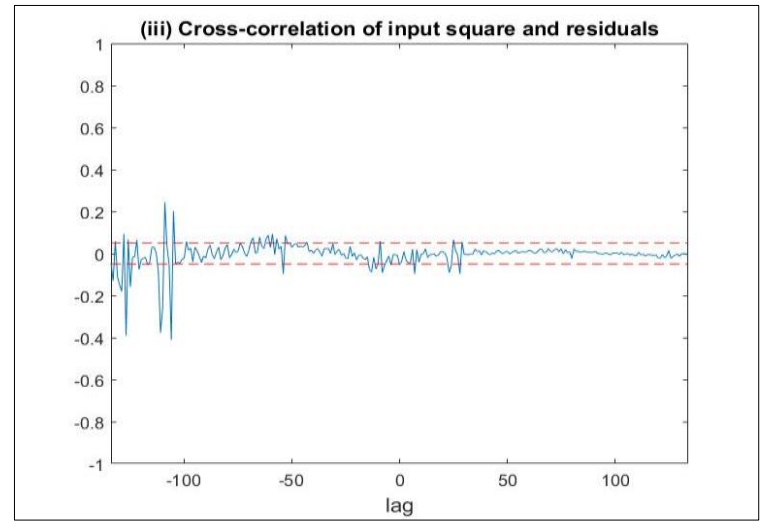

Fig. 5: Validation test with cross-correlation of input square and residuals.

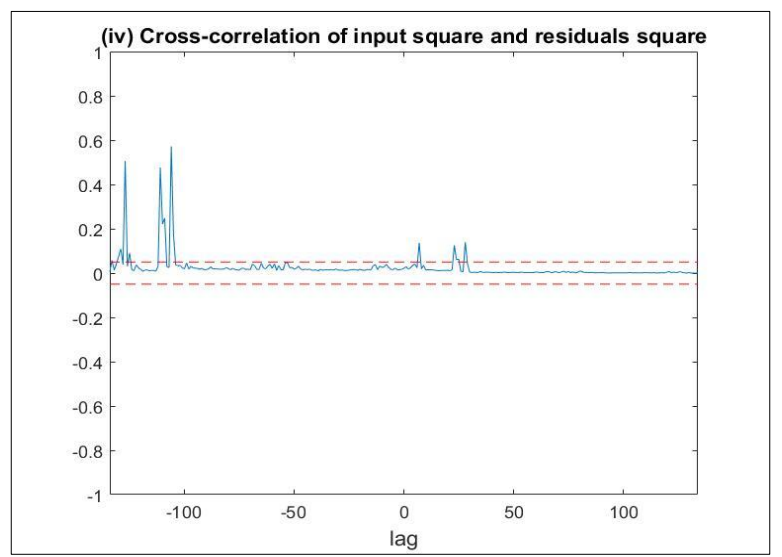

Fig. 6: Validation test with cross-correlation of input square and residuals square.

Auto-correlation shows sharp spike at the center as it was formed by correlating two error signals as shown in Fig. 5. While, the cross-correlation was a correlated signal between error data and normalized input data. From Fig. 6, 7, and 8 shows that there were lines that out of confident line. But, overall correlated signal produced can be considered as stable because just a few signals that stay out of confident line than the signal within the confident line. Hence, the parameter identified is accepted.

The pole-zero plot based on the transfer function obtained. Stability of the system can be determined directly from its transfer function. Hence, the pole-zero map of the parameters identified has been referred to check the stability of the dynamic system model. The zero is located by a circle (o) while pole is located with a cross (x). The pole-zero map of the selected model is shown in Fig. 7.

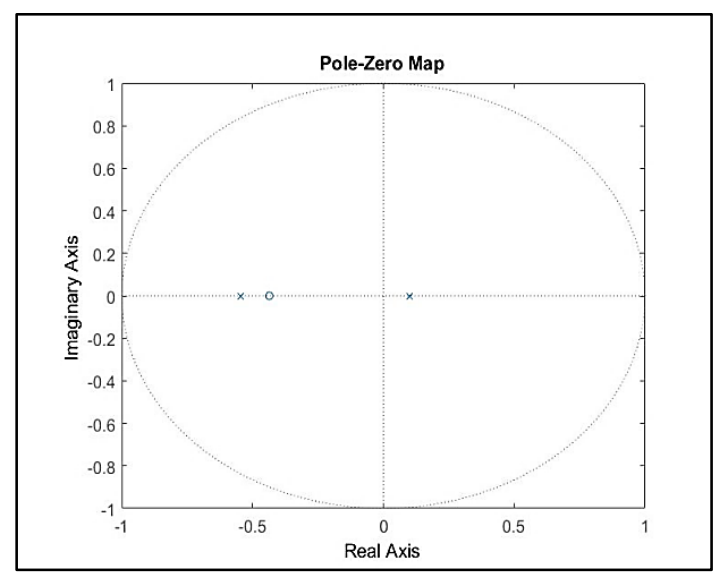

Fig. 7: Pole-zero plot of identified system.

From Fig. 7, it shows that the model selected is unstable as there is a pole lie on the right-half of the s-plane. But, the model is acceptable because the stability of the system can be adjusted by developing the controller from the model obtained. Hence, the model order of 6 with the generation gap of 0.5 is accepted as the best model.

\section{Conclusion}

System identification has been done to construct the dynamic model of the system. All the data acquired is used to develop the ARX model structure. Real-Coded Genetic Algorithm (RCGA) is applied to optimize the objective function in identified the best parameter to create the best model. The performance of toothbrush rig parameter has been analyzed by using different model order. The best model was selected based on a few criteria; the least objective function value, the least standard deviation for 10 times analysis and the fastest convergence time. The model has been validated by correlation analysis and pole-zero map. Hence, the model order 2 had the lowest Mean Square Error (MSE) and standard deviation values. The convergence time can be shorten through the application of generation gap 0.5 instead of 0.9 without loss of model accuracy.

\section{Acknowledgements}

This paper was partly sponsored by the Center for Graduate Studies, Universiti Tun Hussein Onn Malaysia (UTHM). The authors also wish to thank Universiti Tun Hussein Onn Malaysia (UTHM) for providing a software involved in conducting parameter estimation using different model orders in Real-Coded Genetic Algorithm (RCGA)

\section{References}

[1] Abo-Hammour Z, Alsmadi O, Momani S, Arqub OA (2013), A Genetic Algorithm Approach for Prediction of Linear Dynamical Systems. Mathematical Problems in Engineering, 1-12.

[2] Andrijic ZU, Bolf N, Rolich T (2011), Optimizing Configurable Parameters of Model Structure Using Genetic Algorithm. TEDI International Interdisciplinary Journal of Young Scientist from the Faculty of Textile Technology 01, 49-54.

[3] Angelova M \& Pencheva T (2011), Tuning Genetic Algorithm Parameters to Improve Convergence Time. International Journal of Chemical Engineering, 1-7.

[4] Bhuvaneswari NS, Praveena R, Divya R (2012), System Identification and Modelling for Interacting and Non-Interacting Tank Systems using Intelligent Techniques. International Journal of Information Sciences and Techniques (IJIST) 02, 23-37.

[5] Chang WD (2007), Nonlinear System Identification and Control Using a Real-Coded Genetic Algorithm. Applied Mathematical Modelling 31, 541-550.

[6] Cherif I \& Fnaiech F (2015), System Identification with a RealCoded Genetic Algorithm (RCGA). International Journal of Applied Mathematics and Computer Science 25, 863-875.

[7] Ghaffari A, Chaibakhsh A, Parsa H (2007), An Optimization Approach Based on Genetic Algorithm for Modeling Benson Type Boiler. American Control Conference.ACC'07 04, 4860-4865.

[8] Mantri G \& Kulkarni NR (2013), Design and Optimization of PID Controller Using Genetic Algorithm. International Journal of Research in Engineering and Technology 02, 926-930.

[9] Rabbani MJ, Hussain K, Khan A, Ali A (2013), Model Identification and Validation for a Heating System Using MATLAB System Identification Toolbox. IOP Conference Series: Materials Science and Engineering Proceedings of the 1st International Conference on Sensing for Industry, Control, Communications, and Security Technologies, ICSICCST 2013 51, 1-10. 